\title{
OPEN Effect of density, phonon scattering and nanoporosity on the thermal conductivity of anisotropic cellulose nanocrystal foams
}

\author{
Varvara Apostolopoulou-Kalkavoura ${ }^{1,2}{ }^{2}$ Pierre Munier ${ }^{1,2}$, Lukasz Dlugozimaa ${ }^{1}$, \\ Veit-Lorenz Heuthe ${ }^{1}$ \& Lennart Bergström ${ }^{1 凶}$
}

Anisotropic cellulose nanocrystal (CNC) foams with densities between 25 and $130 \mathrm{~kg} \mathrm{~m}^{-3}$ (CNC $_{25}-$ $\mathrm{CNC}_{130}$ ) were prepared by directional ice-templating of aqueous dispersions. Estimates of the solid and gas conduction contributions to the thermal conductivity of the foams using a parallel resistor model showed that the relatively small increase of the radial thermal conductivity with increasing foam density can be attributed to interfacial phonon scattering. The foam wall nanoporosity and, to a lesser extent, the orientation of the CNC particles and alignment of the columnar macropores, also influence the insulation performance of the foams. The insight on the importance of phonon scattering for the thermal insulation properties of nanocellulose foams provides useful guidelines for tailoring nanofibrillar foams for super-insulating applications.

Biobased materials for thermal insulation and thermal management have received substantial research interest thanks to their low thermal conductivity ${ }^{1-3}$ and potential to reduce the carbon footprint by replacing fossilderived insulating materials such as polyurethane and polystyrene foams $\mathrm{s}^{4,5}$. Recent work has also suggested that an increase in the share of biobased materials in buildings could serve as an important carbon sink $\mathrm{k}^{6}$.

Cellulose nanomaterials (CNMs) display a combination of high strength and flexibility ${ }^{7}$ with an anisotropic thermal conductivity ${ }^{8}$, which can be beneficial for, e.g., insulation and thermal management of small devices ${ }^{9}$. While traditional cellulose-based insulation materials, e.g. cavity fillers, are moderately efficient, with thermal conductivities around $40-50 \mathrm{~mW} \mathrm{~m}^{-1} \mathrm{~K}^{-110}$, CNM-based foams and aerogels can display thermal conductivities substantially below the value for air ${ }^{11-15}$. Recent work on ice-templated anisotropic cellulose nanofibril (CNF)-based foams has shown that the thermal conductivity perpendicular to aligned CNFs depends on both the diameter of the particles and the moisture-controlled swelling of the foam walls ${ }^{16}$. Moisture-induced effects are complex and involve both a reduction of the thermal boundary conductance by the increase in the separation distance between the oriented nanofibrils with uptake of water, and an increase in the thermal conductivity when air is replaced with water in the foam walls ${ }^{16}$. Studies on CNM-based isotropic foams and aerogels revealed a complex and non-linear relationship between density and thermal conductivity ${ }^{12,14,17,18}$, which suggests that other parameters related to the structure and morphology of the foams and aerogels, and the nanostructure of the nanofibrillar pore/cell walls, probably have a strong impact on the thermal conductivity. The lack of a detailed structural characterization and relatively narrow explored density ranges in previous studies have impeded a better understanding of the thermal conductivity of CNM foams and aerogels.

Here, we have investigated the density dependence of the thermal conductivity of anisotropic CNC foams, and discussed the heat transfer contributions by including structural and morphological features of the foams in volume-weighted models. The relative importance of foam density, CNC particle orientation, foam wall nanoporosity and macropore alignment are discussed, and phonon scattering at the interfaces between aligned CNCs is identified as the main reason for the low thermal conductivity of the CNC foams perpendicular to the freezing direction.

\footnotetext{
${ }^{1}$ Department of Materials and Environmental Chemistry, Stockholm University, 10691 Stockholm, Sweden. ${ }^{2}$ These authors contributed equally: Varvara Apostolopoulou-Kalkavoura and Pierre Munier. ${ }^{\bowtie}$ email: lennart.bergstrom@ mmk.su.se
} 


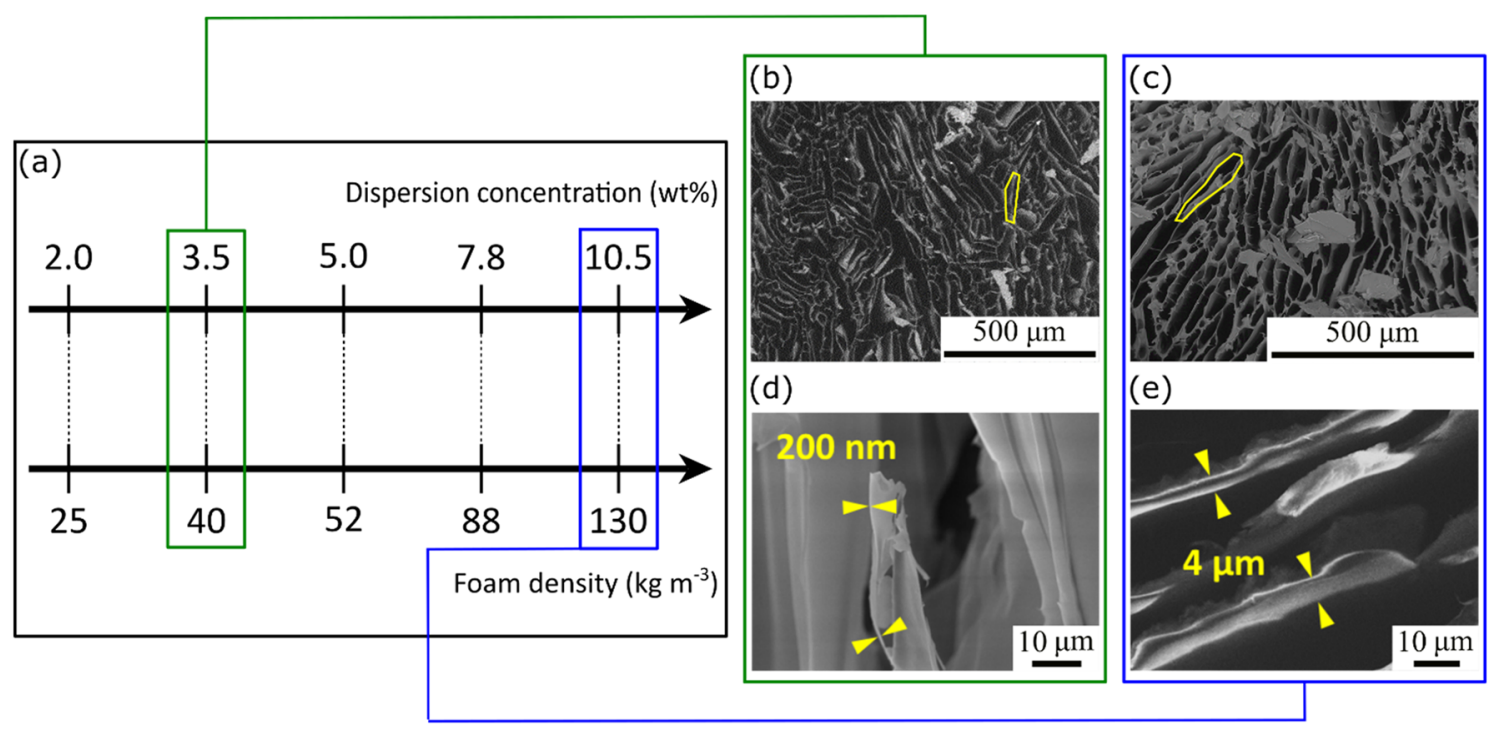

Figure 1. Anisotropic CNC foams prepared by freeze-casting of aqueous dispersions; (a) CNC dispersion concentration and corresponding foam density ranges studied herein. Scanning Electron Microscopy (SEM) images of cross-sections of the columnar macroporous structures for foams with densities of; (b) $40 \mathrm{~kg} \mathrm{~m}^{-3}$; and; (c) $130 \mathrm{~kg} \mathrm{~m}^{-3}$. High-resolution Scanning Electron Microscopy (HRSEM) images of foam walls observed in freeze-cast and freeze-dried foams with densities of; (d) $40 \mathrm{~kg} \mathrm{~m}^{-3}$; and; (e) $130 \mathrm{~kg} \mathrm{~m}^{-3}$. The foam wall thicknesses are indicated by yellow arrows.

\section{Results and discussion}

Preparation, structure and porosity of freeze-cast CNC foams. We have prepared anisotropic foams from cellulose nanocrystal (CNC) dispersions by ice templating, or freeze-casting, followed by freezedrying. CNCs offer a wider dispersion concentration range than for instance CNF dispersions, which gel at relatively low solid contents ${ }^{19}$ and become highly viscous and difficult to process. The density of freeze-cast and freeze-dried foams is directly related to the solid content of the initial liquid dispersions and we have covered a dispersion concentration range from 2.0 to $10.5 \mathrm{wt} \%$ (Fig. 1a). More dilute dispersions do not lead to self-standing foams without the addition of binders or additives, while more concentrated dispersions become difficult to process due to their high viscosity. The correlation between the dispersion concentrations and the foam densities is provided in Supplementary Table S1, and the foams will be referred to, as specified in the right-most column, with an acronym specifying their dry density (in $\mathrm{kg} \mathrm{m}^{-3}$ ).

The foams were produced using CelluForce CNCs, with diameters of $4.3 \pm 0.8 \mathrm{~nm}$ and lengths of $173 \pm 41 \mathrm{~nm}$ (Supplementary Fig. S1), which corresponds to an average aspect ratio around 40. The CNCs possess sulfate half-esters as surface groups and a surface charge of $0.31 \pm 0.01 \mathrm{mmol} \mathrm{OSO}_{3}^{-} \mathrm{g}^{-1}$.

The macropores, i.e. the pores that are enclosed by the ice-templated foam walls, display a columnar form due to the unidirectional ice growth (Supplementary Fig. S2), with elongated cross-sections (Fig. 1b, c). The porosity of the CNC foams, which were gravimetrically determined in a moisture-free atmosphere, ranged from 98.3 down to $91.3 \%$, corresponding to foams with densities of 25 and $130 \mathrm{~kg} \mathrm{~m}^{-3}$, respectively. The foam wall thicknesses increased with increasing density, ranging from a few hundred nanometers for the low density $\left(25-40 \mathrm{~kg} \mathrm{~m}^{-3}\right)$ foams (Fig. 1d) to several micrometers for the high density $\left(130 \mathrm{~kg} \mathrm{~m}^{-3}\right)$ foams (Fig. 1e).

Freeze-casting orients anisotropic particles in the freezing direction ${ }^{20}$, which from here on will be referred to as the axial direction, with the radial direction referring to the direction perpendicular to the freezing direction (Fig. 2a, inset). The foams possess a hierarchical porous structure having not only macropores but also nanopores within the foam walls, as shown by nitrogen sorption measurements (Fig. 2a).

The nanopore volume of the CNC foams increases with increasing density up to a foam density of $34 \mathrm{~kg} \mathrm{~m}^{-3}$ $\left(\mathrm{CNC}_{34}\right.$ foams), followed by a decrease in the nanopore volume with increasing density for denser foams; from $7.5 \mathrm{~mm}^{3} \mathrm{~g}^{-1}$ for $\mathrm{CNC}_{34}$ foams to about $1.6 \mathrm{~mm}^{3} \mathrm{~g}^{-1}$ for $\mathrm{CNC}_{88}$ foams and above. The maximum nanopore volume correlates to the concentration range for the onset of formation of a cholesteric phase in a CNC aqueous dispersion $^{21}$. The decrease in the nanopore volume with increasing foam density can be related to a denser packing of the CNC particles as freeze-casting is performed at dispersion concentrations with increasing amounts of cholesteric phase $\mathrm{e}^{22,23}$. It is interesting to note that even the $\mathrm{CNC}$ foams with the highest nanoporosity, $\mathrm{CNC}_{34}$, display a four times lower nanopore volume compared to freeze-cast CNF foams ${ }^{24}$ where dense packing is hindered by the entanglement of the kinked and flexible nanofibrils.

The orientation of the (partially) crystalline CNC particles in the foams can be estimated by XRD measurements via the extraction of the particle orientation parameter, also called Hermans' orientation parameter $\left(\bar{P}_{2}\right)$. The particle orientation parameter in the CNC foams fluctuates and ranged between $\bar{P}_{2}=0.49$ for foams with an intermediate density $\left(\mathrm{CNC}_{40}\right.$ foams), to $\bar{P}_{2} \geq 0.37$ for foams with higher densities, and $\bar{P}_{2}=0.32$ for the $\mathrm{CNC}_{25}$ 

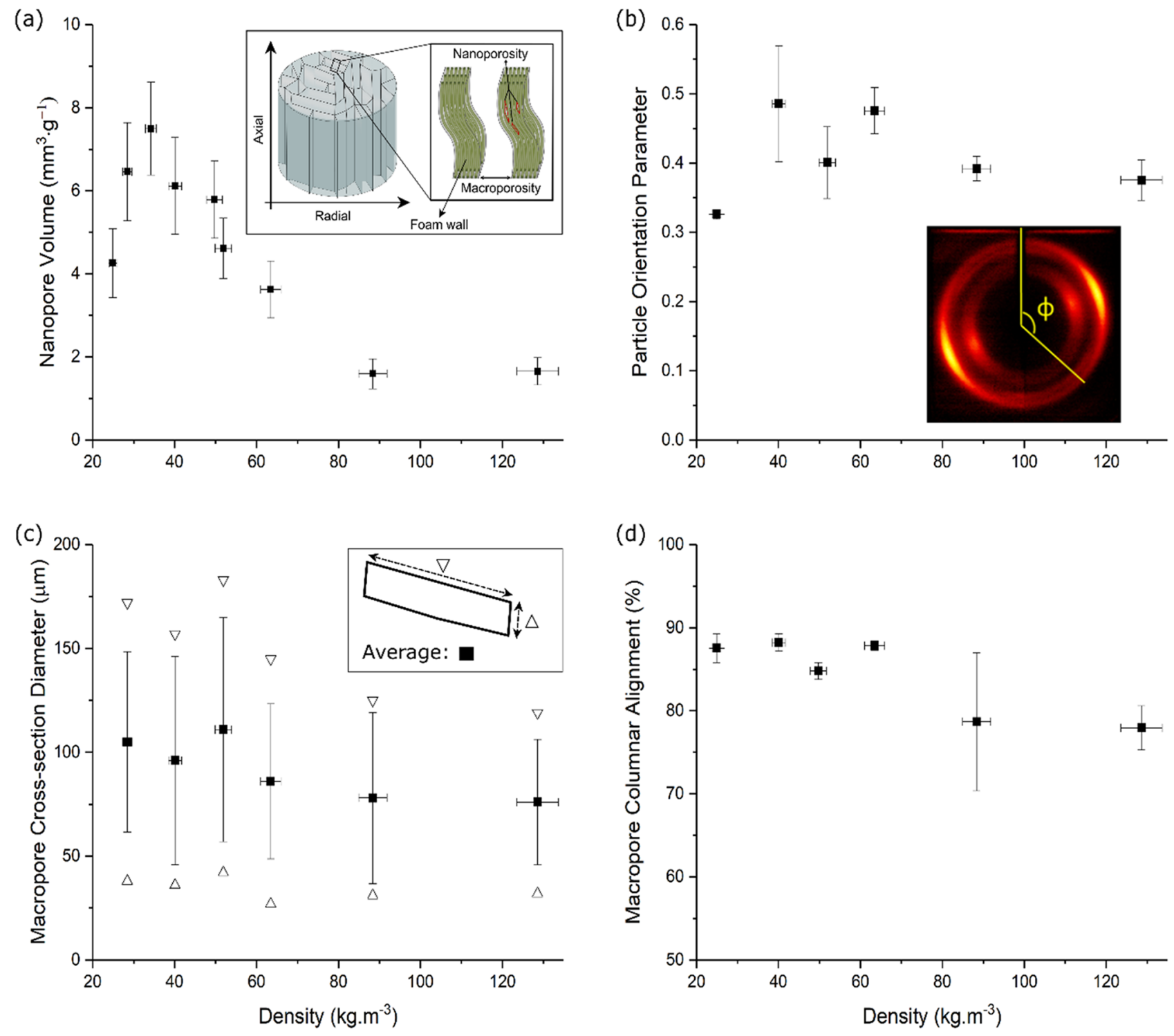

Figure 2. Porosity and alignment in CNC foams. (a) Nanopore volume determined by $\mathrm{N}_{2}$ adsorption isotherms in CNC foam walls as a function of foam density. Inset Schematic illustration of the structure of the anisotropic foam and the foam walls. (b) Particle orientation parameter in CNC foams as a function of foam density. Inset Typical X-ray diffraction (XRD) 2D-pattern of a CNC foam (the angle $\phi$ depicted in the image is defined in the "Methods" section). (c) Macropore cross-section diameter as a function of foam density (the hollow triangles refer to the lengths and widths of the elongated macropore cross-sections, the filled squares refer to the average of both dimensions). (d) Macropore columnar alignment estimated by SEM image analysis as a function of foam density.

foams (Fig. 2b). The low particle orientation for the foam with the lowest density $\left(\mathrm{CNC}_{25}\right)$ and thinnest foam walls may be related to a less effective collective alignment process.

The macropore cross-section diameter (Fig. 2c) was essentially unaffected by the foam density, and the macroporous columnar alignment (Fig. 2d) decreased only slightly with increasing foam density, which suggests that the CNC particles have a minor effect on the growth of the ice crystals during freeze-casting.

Thermal conductivity of CNC foams and volume-weighted estimates. The anisotropic thermal conductivity was measured in a customised Hot Disk setup where the temperature and RH could be controlled ${ }^{25}$.

The relative uncertainty of the radial thermal conductivity $\left(\lambda_{\mathrm{r}}\right)$ values was estimated to be $12 \%$ by a propagation analysis of the uncertainties of the parameters required for their calculation, namely the radial thermal diffusivity $\left(\alpha_{\mathrm{r}}\right)$ (Supplementary Fig. S3), the density $(\rho)$ (Supplementary Table S1) and the specific heat capacity $\left(\mathrm{C}_{\mathrm{p}}\right)$ (Supplementary Fig. S4), following Eq. (1):

$$
\lambda_{r}=\alpha_{r} \rho C_{p}
$$



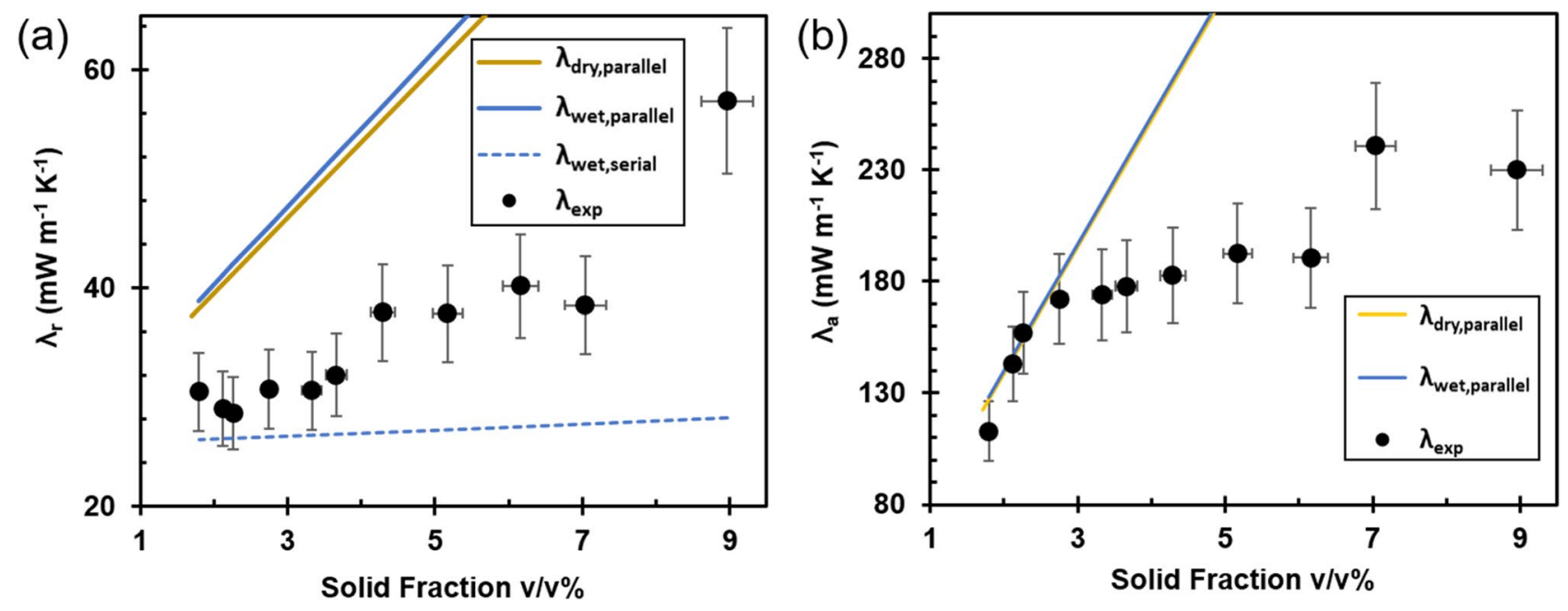

Figure 3. Thermal conductivity of CNC foams as a function of solid fraction (v/v\%). (a) Radial $\left(\lambda_{\mathrm{r}}\right)$ and; (b) axial $\left(\lambda_{\mathrm{a}}\right)$ thermal conductivity of CNC foams as a function of CNC solid fraction (v/v\%) at $295 \mathrm{~K}$ at $50 \% \mathrm{RH}$. The black filled circles correspond to experimental data, while the solid lines correspond to volume-weighted parallel sum-based theoretical estimates of the thermal conductivities, at; (i) dry $\left(\lambda_{\text {dryparallel }}\right)$ and (ii) $50 \% \mathrm{RH}$ wet conditions $\left(\lambda_{\text {wet,parallel }}\right)$, and the dotted line corresponds to the serial addition for $50 \% \mathrm{RH}$ wet conditions $\left(\lambda_{\text {wet,serial }}\right)$.

The propagation of uncertainly follows Eq. (2):

$$
\Delta \lambda_{r}=\lambda_{r} \sqrt{\left(\frac{\Delta \alpha_{r}}{\alpha_{r}}\right)^{2}+\left(\frac{\Delta \rho}{\rho}\right)^{2}+\left(\frac{\Delta C_{p}}{C_{p}}\right)^{2}}
$$

where $\Delta X$ is the total uncertainty of the $\mathrm{X}$ variable, which is a sum of the random and systematic uncertainties ${ }^{26}$. The random uncertainties of $\alpha_{r}, \rho$ and $C_{p}$ were based on estimates of the average relative standard deviations (SD) obtained from repeated measurements on several specimens (at least four per specimen for $\rho$, at least five per specimen for $\alpha_{r}$ and five in total for $C_{p}$ ), and were multiplied with 1.65 that relates to a $95 \%$ confidence interval $^{26}$. The obtained relative random uncertainties were $6 \%, 4 \%$ and $1 \%$ for $\alpha_{r}, \rho$ and $C_{p}$, respectively. The systematic uncertainty or instrumental bias of $\alpha_{r}$ was estimated to be $5 \%{ }^{27}$, while no systematic uncertainty was considered for $\rho$ and $C_{p} . \Delta C_{p}$ also incorporates the uncertainty on the measurement of water uptake at different $\mathrm{RH}$, which was used to determine $\mathrm{C}_{\mathrm{p}}$ at various $\mathrm{RH}$ using the rule of mixtures.

The density dependence of the radial thermal conductivity $\left(\lambda_{\mathrm{r}}\right)$ and the axial thermal conductivity $\left(\lambda_{\mathrm{a}}\right)$ of CNC foams, at $50 \% \mathrm{RH}$, are shown in Fig. 3. The $\lambda_{\mathrm{r}}$, perpendicularly to the oriented fibrils (Fig. 3a), was between four and six times smaller than the $\lambda_{\mathrm{a}}$ (Fig. $3 \mathrm{~b}$ ). The $\lambda_{\mathrm{r}}$ was relatively unaffected by RH but the $\lambda_{\mathrm{a}}$ increased with increasing RH (Supplementary Fig. S5). The main part of the analysis and discussion will be devoted to the radial thermal conductivity at 50\% RH but similar trends are observed at 5, 20 and $80 \%$ RH (Supplementary Fig. S6).

Figure 3 a shows that, at $50 \% \mathrm{RH}, \lambda_{\mathrm{r}}$ remained in the range of $28-32 \mathrm{~mW} \mathrm{~m}^{-1} \mathrm{~K}^{-1}$ for foams with dry solid fractions between 1.7 and 3.5\%, which corresponds to dry densities between 25 and $52 \mathrm{~kg} \mathrm{~m}^{-3}$ (see Supplementary Table S1), and increased with increasing solid fraction for foams with dry solid fractions above $3.5 \%$, up to $57 \mathrm{~mW} \mathrm{~m}^{-1} \mathrm{~K}^{-1}$ for the $\mathrm{CNC}$ foams with the highest solid fraction $\left(\mathrm{CNC}_{130}\right.$ foams). The axial thermal conductivity, $\lambda_{\mathrm{a}}$, displayed a pronounced increase with increasing solid fraction for low solid fraction foams (up to $\mathrm{CNC}_{40}$ foams), followed by a less steep increase for the high solid fraction foams (Fig. 3b).

The thermal conductivity of the porous foams has been estimated by the so-called parallel resistor model with volume fraction-weighted sums of the solid and gas contributions to the heat transport. The parallel resistor model assumes a simultaneous (parallel) heat transfer through the solid and gas phase ${ }^{28}$. The foam walls of the CNC foams provide connected solid conduction pathways in both the axial and radial directions (see inset in Fig. 2a), which suggests that the parallel resistor model is appropriate to model both the radial and axial thermal conductivities. The radiation and convection contributions are expected to be insignificant as the temperature is relatively low $(295 \mathrm{~K})$, and the pore size of the foams is smaller than $1 \mathrm{~mm}$, respectively. The foam walls were considered to consist of densely packed CNC particles that are fully aligned in the axial direction. The thermal conductivity of CNC particles $\left(\lambda_{\text {cell }}\right)$, perpendicular to $\left(720 \mathrm{~mW} \mathrm{~m}^{-1} \mathrm{~K}^{-1}\right)$ and along $\left(5700 \mathrm{~mW} \mathrm{~m}^{-1} \mathrm{~K}^{-1}\right)$ their long axis were used as an approximation of the solid contribution in the radial and axial direction, respectively ${ }^{8}$. The thermal conductivity of air at $295 \mathrm{~K}\left(\lambda_{\text {air }}=25.7 \mathrm{~mW} \mathrm{~m}^{-1} \mathrm{~K}^{-1}\right)$ was used for the gas contribution. Using the parallel resistor model, the thermal conductivity for foams with dense walls at dry conditions, $\lambda_{\text {dry }}$ can be expressed as:

$$
\lambda_{\text {dry,parallel }}=\phi_{\text {air }} \cdot \lambda_{\text {air }}+\phi_{\text {cell }} \cdot \lambda_{\text {cell }}
$$


where $\phi_{\text {air }}$ and $\phi_{\text {cell }}$ are the volume fractions of air and foam walls, respectively, normalized to the total apparent volume of the foam $\left(\phi_{\text {air }}+\phi_{\text {cell }}=1\right)$. $\phi_{\text {air }}$ and $\phi_{\text {cell }}$ were estimated from the apparent density of the foam and the skeletal density of cellulose (see "Materials and methods").

CNC foams take up water and the contribution of the water content at 50\% RH (Supplementary Fig. S7) with $\lambda_{\mathrm{H} 2 \mathrm{O}}=600 \mathrm{~mW} \mathrm{~m}^{-1} \mathrm{~K}^{-1}$ was included in the estimate of the thermal conductivity of moisture-containing foams $\left(\lambda_{\text {wet }}\right)$ by Eq. (4):

$$
\lambda_{\text {wet,parallel }}=\phi_{\text {air }} \cdot \lambda_{\text {air }}+\phi_{\text {cell }} \cdot \lambda_{\text {cell }}+\phi_{\mathrm{H} 2 \mathrm{O}} \cdot \lambda_{\mathrm{H} 2 \mathrm{O}}
$$

where $\phi_{\mathrm{H} 2 \mathrm{O}}$ is the water volume fraction, obtained from the gravimetrically determined water uptake (see "Materials and methods"). It is interesting to note that the difference between $\lambda_{\text {dry }}$ and $\lambda_{\text {wet }}$ direction is (1-2 $\mathrm{mW} \mathrm{m}^{-1} \mathrm{~K}^{-1}$ ), which suggests that the replacement of air with water has a minor influence on the thermal conductivity of $\mathrm{CNC}$ foams at $50 \% \mathrm{RH}$.

The thermal conductivity can also be described by the serial resistor model ${ }^{29-31}$. Combinations of the parallel model (described above) and the serial model have been used to fit the thermal conductivity of various isotropic porous materials ${ }^{31-33}$. The serial model involves addition of the different contributions to the thermal conductivity at $50 \% \mathrm{RH}$ as given by Eq. (5):

$$
\lambda_{\text {wet }, \text { serial }}=\frac{1}{\frac{\phi_{\text {air }}}{\lambda_{\text {air }}}+\frac{\phi_{\text {cell }}}{\lambda_{\text {cell }}}+\frac{\phi_{H 2 O}}{\lambda_{H 2 O}}}
$$

The serial model, as it assumes a heat transfer pathway that alternates from the solid to the gas phases, results in much lower values compared to the parallel model (Fig. 3a).

Figure $3 \mathrm{~b}$ shows that the volume-weighted estimates of the gas and solid contributions using the parallel resistor model, Eqs. (3) and (4), corresponded relatively well to the axial thermal conductivity of CNC foams up to a solid fraction of $3.3 \%$, or a density of $50 \mathrm{~kg} \mathrm{~m}^{-3}$, but overestimates the axial thermal conductivity at high foam solid fractions. It should be noted that a reduction of the assigned value of $5.7 \mathrm{~W} \mathrm{~m}^{-1} \mathrm{~K}^{-1}$ for the solid phase thermal conductivity of cellulose in the axial direction ${ }^{8}$ would improve the fit between the theoretical estimate and the experimental values also for the high solid fraction foams but the non-linear solid fractions dependence of $\lambda_{\mathrm{a}}$ suggests that there are other factors than a possible reduction of the solid phase thermal conductivity that contribute to limit the increase of $\lambda_{\mathrm{a}}$ with increasing density.

Knudsen effect and phonon scattering. It is well known that the gas conduction is significantly reduced when the pore size becomes similar to or smaller than the mean free path of air molecules, the so-called Knudsen effect ${ }^{34}$. The effect of nanopores on the gas conduction contribution to thermal conductivity, $\lambda_{\mathrm{np}}$, can be estimated by Eq. (6):

$$
\lambda_{\mathrm{np}}=\frac{\lambda_{\text {air }}}{1+2 \beta \cdot \mathrm{Kn}}
$$

where $\beta$ is a characteristic number equal to 2 for foams and aerogels and $\mathrm{Kn}$ is the Knudsen number, which can be estimated by dividing the mean free path of air molecules by the pore $\operatorname{size}^{34}$. The nanoporosity of the foam walls at $50 \% \mathrm{RH}$ varied between 5 and $8 \%$ (this range varies at other RH due to different swelling percentages, see Supplementary Fig. S7), and the average nanopore diameters ranged between 7 and $10 \mathrm{~nm}$ (Supplementary Table S2).

The high Knudsen number (4-6) in the nanopores results in a $\lambda_{\text {np }}$ that is below $1.5 \mathrm{~mW} \mathrm{~m}^{-1} \mathrm{~K}^{-1}$ at $0-80 \% \mathrm{RH}$ for all CNC foams, while thermal conductivity in the much larger macropores, $\lambda_{\mathrm{mp}}$ (calculated with the same formula), is very close to the value for air because the Knudsen effect is negligible at pore sizes above $30 \mu \mathrm{m}$ (Fig. 2c).

By incorporating the Knudsen effect and introducing separate gas contributions for the macropores $\left(\lambda_{\mathrm{mp}}\right)$ and the nanopores $\left(\lambda_{\mathrm{np}}\right)$, we obtain a parallel volume-weighted estimate of the radial thermal conductivity of moisture-containing foams, $\lambda_{\text {wet,Kn,parallel }}$ expressed by Eq. (7):

$$
\lambda_{\text {wet }, \text { Kn,parallel }}=\phi_{m p} \cdot \lambda_{m p}+\phi_{n p} \cdot \lambda_{n p}+\phi_{\text {cell }} \cdot \lambda_{\text {cell }}+\phi_{H 2 O} \cdot \lambda_{H 2 O}
$$

where $\phi_{n p}$ is estimated from the nanopore volume obtained by $\mathrm{N}_{2}$ adsorption measurements (see Fig. 2a) and the total foam volume, while $\phi_{m p}$ corresponds to the remaining volume of air $\left(\phi_{m p}+\phi_{n p}=\phi_{a i r}\right)$. However, the $\lambda_{\text {wet,Kn,parallel }}$ estimate is much higher than the measured radial thermal conductivity (Fig. 4), which shows that the Knudsen effect is of minor importance due to the small fraction of nanopores in the foams. Nevertheless, $\lambda_{\mathrm{r}}$ attained a value close to the value for air at $\Pi_{\mathrm{np}}$ values above $7 \%$ at $50 \% \mathrm{RH}$, (Supplementary Fig. S8).

The solid conduction contribution to the thermal conductivity of nanostructured materials can be substantially reduced by phonon scattering at particle-particle interfaces ${ }^{16}$. The effect of phonon scattering at interfaces can be expressed by the interfacial thermal resistance, or Kapitza resistance $\left(R_{k}\right)$, which can be estimated by Eq. (8):

$$
R_{k}=\frac{g_{i}}{\lambda_{i}}=\frac{d_{t}}{\lambda_{t}}-2 \cdot \frac{d}{\lambda_{\text {cell }}}
$$

where $\mathrm{g}_{\mathrm{i}}$ is the surface separation distance between two adjacent CNC particles, $\lambda_{\mathrm{i}}$ is the interfacial thermal conductivity, $d$ is the average diameter of a CNC particle $(=4.3 \pm 0.8 \mathrm{~nm})$, and $\lambda_{\mathrm{t}}$ and $\mathrm{d}_{\mathrm{t}}$ are the thermal conductivity and length, respectively, of a system consisting of two CNC particles placed parallel to each other with a gap, $g_{i}$. The interfibrillar separation distance $g_{i}$ has been shown to depend on the water uptake and was 


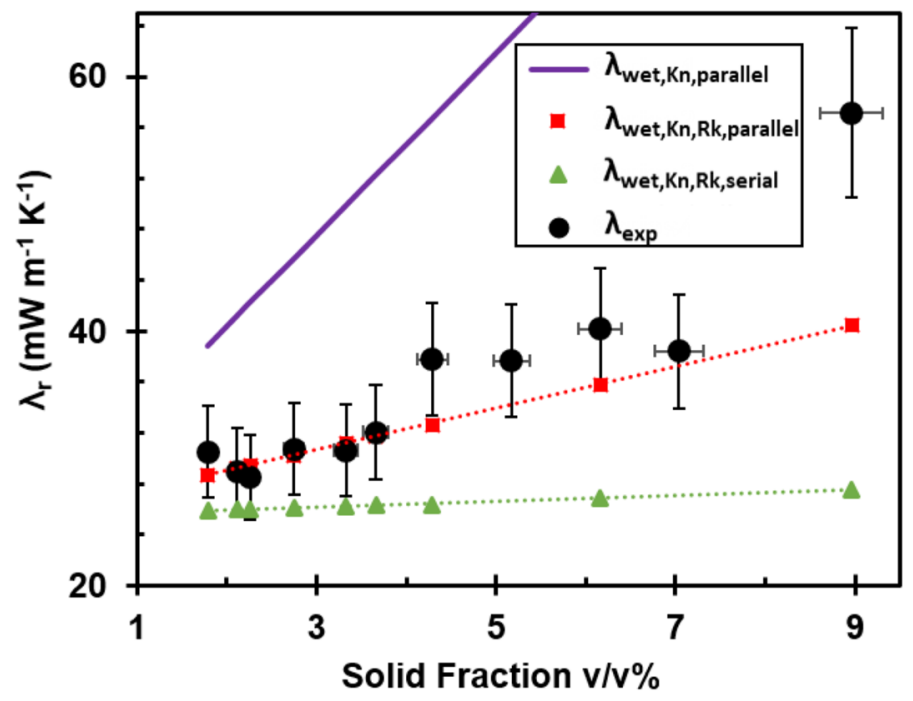

Figure 4. Solid fraction-dependent heat transfer mechanisms in CNC foams. Radial thermal conductivity $\left(\lambda_{\mathrm{r}}\right)$ of CNC foams as a function of CNC solid fraction (v/v\%) including the experimental data at $50 \% \mathrm{RH}$, the theoretical estimates of $\lambda_{\text {wet,Kn,parallel }}$ including both the uptake of water at $50 \% \mathrm{RH}$ and the Knudsen effect in the nanopores, and the theoretical estimates of $\lambda_{\text {wet,Kn,Rk,parallel }}$ and $\lambda_{\text {wet,Kn,Rk,serial }}$ additionally including the particleparticle interfacial effects in the foam walls and distinguishing between parallel and serial addition.

estimated to vary between 5.1 and $6.2 \AA$ in the foam walls of freeze-cast CNF foams for RH $35-65 \%{ }^{16}$. The water uptake of CNC foams is significantly smaller than for CNF foams ${ }^{16}$ and we have used the calculated value of $g_{i}$ of $2.3 \pm 0.4-3.7 \pm 0.7 \AA$ at $50 \%$ RH (see Supplementary Fig. S7). The value for $\lambda_{\mathrm{t}}=270 \mathrm{~mW} \mathrm{~m}^{-1} \mathrm{~K}^{-1}$ was obtained from Diaz et $\mathrm{al}^{8}$. The interfacial thermal resistance in the radial direction of aligned CNC particles was estimated to $2.2 \times 10^{-8} \mathrm{~m}^{2} \mathrm{~K} \mathrm{~W}^{-1}$. It is interesting to note that the estimated $\mathrm{R}_{\mathrm{k}}$ for CNC is of a similar magnitude $\left(10^{-8} \mathrm{~m}^{2} \mathrm{~K} \mathrm{~W}^{-1}\right)$ as for carbon nanotubes ${ }^{35}$.

The effect of phonon scattering at interfaces can be included in an estimate of the (solid) thermal conductivity of a thin film (i.e. the foam wall) of aligned CNC nanoparticles, $\lambda_{\mathrm{p}}$, by Eq. (9):

$$
\lambda_{p}=\frac{\lambda_{\text {cell }}}{1+\lambda_{\text {cell }} \cdot \frac{R_{k}}{d}}
$$

where $\lambda_{\text {cell }}$ is the radial thermal conductivity of a single CNC particle $\left(720 \mathrm{~mW} \mathrm{~m}^{-1} \mathrm{~K}^{-1}\right.$ as mentioned above $\left.\mathrm{e}^{8}\right)$. Including the estimated interfacial thermal resistance for the $\mathrm{CNC}$ foam walls $\left(2.2 \times 10^{-8} \mathrm{~m}^{2} \mathrm{~K} \mathrm{~W}^{-1}\right)$ resulted in an estimated solid contribution to the thermal conductivity in the radial direction, $\lambda_{\mathrm{p}}$, of $158-163 \mathrm{~mW} \mathrm{~m}^{-1} \mathrm{~K}^{-1}$ at $50 \% \mathrm{RH}$. The parallel volume-weighted estimate for the radial thermal conductivity of moisture-containing foams that accounts for both phonon scattering and Knudsen effects, $\lambda_{\text {wet,Kn,Rk,parallel }}$ is given by Eq. (10):

$$
\lambda_{\text {wet }, \mathrm{Kn}, \mathrm{R}, \text { parallel }}=\phi_{m p} \cdot \lambda_{m p}+\phi_{n p} \cdot \lambda_{n p}+\phi_{\text {cell }} \cdot \lambda_{p}+\phi_{\mathrm{H} 2 \mathrm{O}} \cdot \lambda_{\mathrm{H} 2 \mathrm{O}}
$$

Figure 4 shows that the obtained $\lambda_{\text {wet,Kn,Rk }}$ estimate corresponds well with the experimental values for the radial thermal conductivities of the $\mathrm{CNC}_{25-88}$ foams, which suggests that a significant reduction of the solid conduction by phonon scattering is essential to obtain anisotropic $\mathrm{CNC}$ foams with low radial thermal conductivities.

For comparison, we have also estimated the thermal conductivity using the corresponding serial resistor model, given by Eq. (11):

$$
\lambda_{\text {wet }, \text { Kn,Rk,serial }}=\frac{1}{\frac{\phi_{m p}}{\lambda_{m p}}+\frac{\phi_{n p}}{\lambda_{n p}}+\frac{\phi_{\text {cell }}}{\lambda_{p}}+\frac{\phi_{H 2 O}}{\lambda_{H 2 O}}}
$$

which shows that the serial model underestimates the radial thermal conductivity (Fig. 4). Phonon scattering at the solid-gas interfaces ${ }^{8,36,37}$ could also reduce the thermal conductivity, but it was not possible to evaluate the possible magnitude of this phenomenon.

\section{Conclusions}

The thermal conductivity of freeze-cast anisotropic $\mathrm{CNC}$ foams with densities between 25 and $130 \mathrm{~kg} \mathrm{~m}^{-3}$ were 4-6 times lower perpendicular to (radially) compared to along (axially) the freezing direction. Theoretical estimates based on the main heat transfer contributions to solid conduction, gas conduction and water uptake using a parallel resistor model, showed that the reduction of the solid conduction due to phonon scattering is much more important than the reduction of the gas contribution by the Knudsen effect for reaching radial thermal conductivities as low as $29 \mathrm{~mW} \mathrm{~m}^{-1} \mathrm{~K}^{-1}$, for the $\mathrm{CNC}_{34}$ foams at $50 \% \mathrm{RH}$ and $295 \mathrm{~K}$ (Fig. 5). The theoretical 


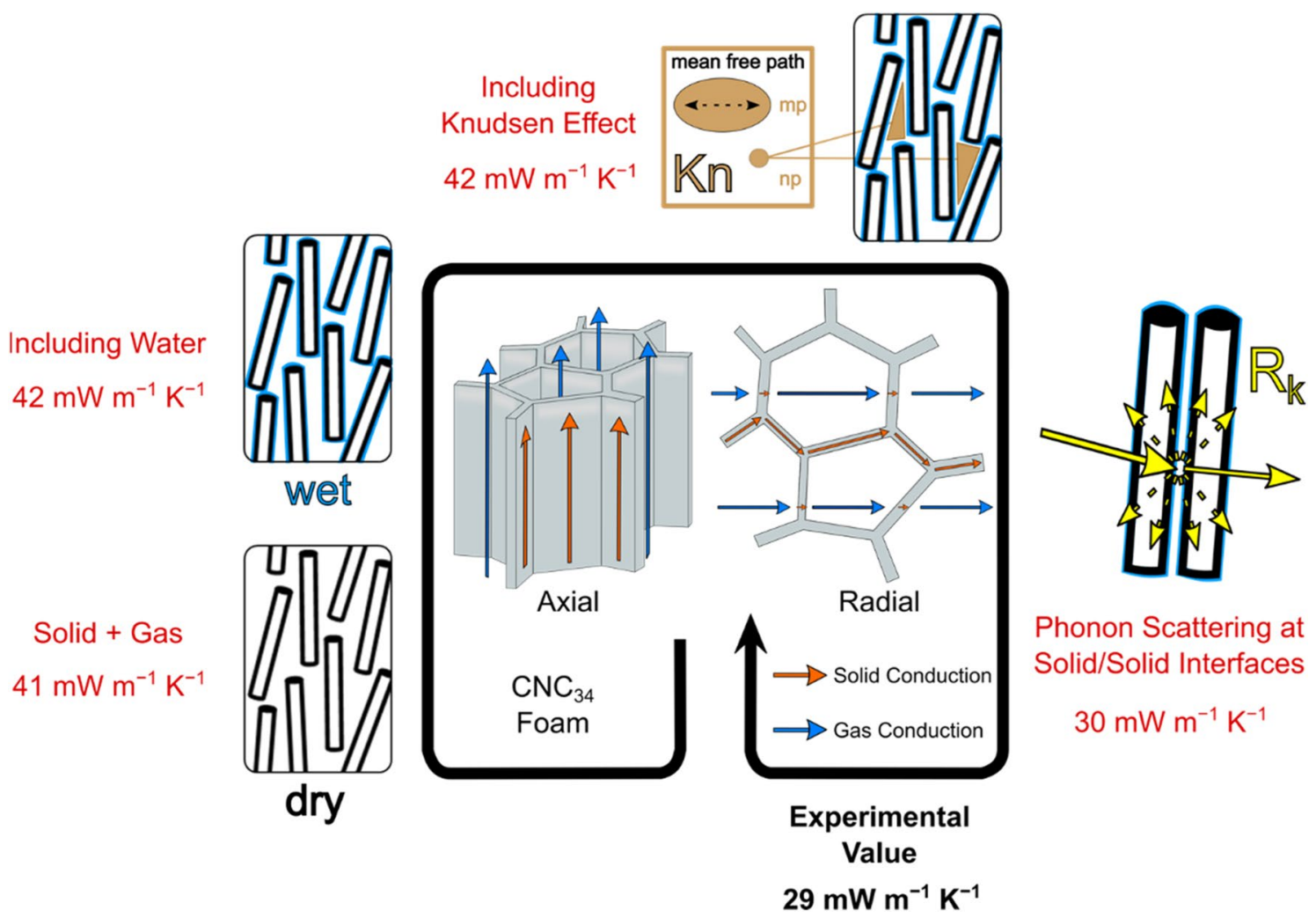

Figure 5. Summary of the assessed heat transfer modes in anisotropic $\mathrm{CNC}$ foams. Illustration of the morphological and structural features and the theoretical estimates (red numbers) based on the summation of the solid and gas contributions to the radial thermal conductivity of $\mathrm{CNC}_{34}$ foams. The experimentally determined radial thermal conductivity is highlighted in black and bold.

estimates suggest that the presence of water and the nanoporosity have a relatively small influence on the radial thermal conductivity. The orientation of the CNC particles, the alignment of the columnar macropores and the macropore size may also influence the axial and radial thermal conductivities of high density foams.

This study shows that phonon scattering dominates the heat transfer properties of CNC foams, and suggests how nanofibrillar foams with high interfacial thermal resistance and super-insulating properties can be prepared. Additional information regarding phonon scattering at solid-solid and solid-air interfaces are needed for accurate prediction of the insulating performance of biobased, nanofibrillar foams and aerogels.

\section{Methods}

Preparation of aqueous dispersions. The CNCs used in this study are a commercial product from CelluForce. The CelluForce powder was dispersed as received in DI water using a mechanical stirrer. The initial concentration was $4.5 \mathrm{wt} \%$ and the dispersion was later either diluted with DI water or upconcentrated using a rotary evaporator device in order to obtain higher concentrations.

Preparation of anisotropic foams. Anisotropic CNC foams were prepared by unidirectional ice templating ${ }^{20,38}$ from dispersions having concentrations of 2, 2.5, 3, 3.5, 4, 4.5, 5, 6, 7, 7.8, 8.9 and $10.5 \mathrm{wt} \%$. Teflon molds, with copper bottom plate, $4 \mathrm{~cm}$ in diameter and $2.5 \mathrm{~cm}$ in height were filled with CNC dispersion and placed in contact with dry ice, giving a cooling rate of $3 \mathrm{~K} \mathrm{~min}^{-1}$. The final dry foams were obtained by ice sublimation at 0.024 mbar and room temperature (RT) for four days using a freeze-dryer (Christ Alpha 1-2LDplus, Germany).

Particle characterization. AFM (Dimension 3100, Bruker, USA) operated in tapping mode was used to determine the CNC particle dimensions (Supplementary Fig. S1). A droplet of $0.001-0.005 \mathrm{wt} \%$ aqueous CNC dispersion was deposited onto freshly cleaved mica substrate and dried at RT.

The charge density (CD) of the CNCs was determined by conductometric titration to be $0.31 \pm 0.01 \mathrm{mmol}$ $\mathrm{OSO}_{3}^{-}$per gram of cellulose for the $\mathrm{CNCs}^{39}$.

Foams characterization. SEM images of the foams cross-sections were taken using a HITACHI TM-3000 (Germany) using a $5 \mathrm{kV}$ or $15 \mathrm{kV}$ electron beam at magnifications of $\times 100-250$. High-resolution SEM images of the foams wall were taken using a JEOL JSM-7000 (USA) and a $15 \mathrm{kV}$ electron beam at a magnification of $\times 50-750$. 
The cross-section diameters of macropores were measured manually using ImageJ on SEM pictures. The local degree of orientation of the columnar macropores was determined by SEM image analysis using the ImageJ plugin "Orientation $\mathrm{J}^{40}$. The image analysis calculates the structure tensor of each pixel of coordinates (x; y) in its local neighborhood. The local orientation and isotropy, i.e., coherency and energy, respectively, of every pixel in the image is evaluated by computing the spatial derivatives in $\mathrm{x}$ and $\mathrm{y}$ directions using a Gaussian gradient. Histograms of the local orientation weighed by the coherency were obtained for each image and they were fitted to a Gaussian function for assessing the orientation index, $\mathrm{f}$. $\mathrm{f}$ was calculated using the full width at half-maximum (fwhm) of the Gaussians as shown in Eq. (12):

$$
\mathrm{f}=\frac{180-f w h m}{180}
$$

The apparent density, $\rho_{a p p}$, of the foams was calculated from the mass and the volume (height $\left.{ }^{*} \pi r^{2}\right)$ of the foams, that had been kept for 3 days at $50 \%$ RH and $295 \mathrm{~K}$.

The foam porosity ( $\left.\phi_{\text {air }}\right)$ was determined from the skeletal density of cellulose $\left(\rho_{\text {skel }}\right)$, chosen as $1500 \mathrm{~kg} \mathrm{~m}^{-338}$, and the apparent foam density $\left(\rho_{a p p}\right)$, according to Eq. (13):

$$
\phi_{\text {air }}=1-\frac{\rho_{a p p}}{\rho_{\text {skel }}}
$$

Nitrogen sorption measurements were performed using ASAP 2020 (Micromeritics Instrument Corporation, Nocross, GA, USA). The CNC foams were degassed at $80^{\circ} \mathrm{C}$ for $10 \mathrm{~h}$. The adsorption and desorption cumulative volume of nanopores (for diameters between 17 and $3000 \AA$ ) and average nanopore diameters were estimated using the Barrett-Joyner-Halenda (BJH) model $^{41}$.

XRD was used to estimate the crystallinity index and the Hermans' orientation parameter of CNCs in the foams walls.

The crystallinity index was calculated from 1D diffractograms (Supplementary Fig. S9) obtained with a Panalytical X'Pert PRO diffractometer operated with $\mathrm{Cu}$ Ka radiation and in Bragg-Brentano diffraction geometry. Pressed foam samples with a mean thickness under $1 \mathrm{~mm}$ were mounted on a Si wafer zero-background holder spinning at a constant rate of $30 \mathrm{rpm}$ and diffraction patterns were measured in a $2 \theta$ range $5^{\circ}-50^{\circ}$ with $0.0167^{\circ}$ step size. The $1 \mathrm{D}$ diffractograms were obtained by integrating the $2 \mathrm{D}$ patterns using the Segal $\operatorname{method}^{42}$ as described in Eq. (14):

$$
C I(\%)=\frac{I_{200}-I_{a m}}{I_{200}} \times 100
$$

where $I_{200}$ and $I_{a m}$ are the intensity values for the (200) peak of crystalline cellulose located at $2 \theta=22^{\circ}-23^{\circ}$ and the amorphous background located at $2 \theta=18^{\circ}-19^{\circ}$, respectively. Angular values measured with the Mo source were converted to those that would be obtained with a copper $(\mathrm{Cu}) \mathrm{X}$-ray source.

For the Hermans' orientation parameter, a Bruker D8 Venture single-crystal diffractometer equipped with a Photon II detector was used with $\mathrm{Cu}-\mathrm{Ka}$ radiation, $100 \mathrm{~mm}$ detector distance and $180 \mathrm{~s}$ acquisition times. The Hermans' orientation parameter of the CNCs within the foams was estimated from 2D XRD patterns obtained from uncompressed pieces of foam. The Hermans' orientation parameter, $\bar{P}_{2}$, quantitatively describes the alignment of the CNCs relatively to the freezing direction. $\bar{P}_{2}$ is obtained by azimuthal integration of the (200) peak of cellulose $\left(\theta=11.4^{\circ}\right)$ and Eqs. (15) and (16):

$$
\begin{gathered}
I(\phi) \cong \sum_{n=0}^{2} a_{n} P_{2 n}(\cos \phi) \\
\bar{P}_{2}=\frac{a_{1}}{5}
\end{gathered}
$$

Equation (15) is a Legendre series expansion with which the azimuthal integration was fitted. $\phi$ represents a theoretical angle between a nanoparticle's main direction and the freezing direction of ice crystals during ice templating. This angle can be identified as the azimuthal angle on the $2 \mathrm{D}$ XRD pattern. $I(\phi)$ represents the intensity of the XRD signal at a certain $\phi$ angle. $P_{2 n}$ are even Legendre polynomials and $a_{n}$ are the corresponding fitting coefficients. After normalizing the fitting coefficients to $a_{0}=1$, the Hermans' orientation parameter is obtained from the $a_{1}$ coefficient according to Eq. $(16)^{43}$.

DSC (Mettler Toledo 820, Sweden) was used to estimate the specific heat capacity at constant pressure $\left(C_{p}\right)$ (Supplementary Fig. S4) of CNC foams at $\mathrm{RH}=0$ under nitrogen atmosphere at temperatures ranging between -20 and $50{ }^{\circ} \mathrm{C}$ with a heating rate of $10 \mathrm{~K} \mathrm{~min}^{-1}$. Aluminum crucibles and lids were used for the DSC measurements. An empty crucible with lid served as the reference, and sapphire was used as a standard. Five independent samples of 8-10 $\mathrm{mg}$ of $\mathrm{CNC}$ obtained by compressing the foams into the crucibles were performed at the DSC resulting in the average $\mathrm{C}_{p}$ value of $898 \pm 6.2 \mathrm{~J} \mathrm{~kg}^{-1} \mathrm{~K}^{-1}$. All samples were carefully dried prior to the addition in the crucibles by heating at $105^{\circ} \mathrm{C}$ for $24 \mathrm{~h}$ and after placing them in the crucibles for 24 additional hours at $105^{\circ} \mathrm{C}$.

The foam wall nanoporosity $\left(\Pi_{n p}\right)$ was estimated using Eq. (17):

$$
\Pi_{n p}=1-\frac{\rho_{\text {wall }}}{\rho_{\text {scel }}}
$$


where $\rho_{\text {wall }}$ is the density of the foam wall and $\rho_{\text {scel }}$ is the skeletal density of cellulose $\left(1500 \mathrm{~kg} / \mathrm{m}^{338}\right)$. The density of the foam wall is estimated by Eq. (18):

$$
\rho_{\text {wall }}=\frac{m_{\text {wet }}}{V_{\text {foam }} \cdot\left(1-\phi_{\text {air }}\right)+V_{n p}}
$$

where $m_{\text {wet }}$ is the $R H$-dependent mass of the foam, $V_{\text {foam }}$ is the volume of the foam, $\phi_{\text {air }}$ is the total porosity of the foam, and $V_{n p}$ is the nanopore volume in the foam walls. The normalized nanopore volume was calculated by multiplying the nanopore volume measured by nitrogen sorption at $R H=0$ with the weight of the foam for the specific foams (Fig. 2a).

The $C_{p}$ of moist foams (Eq. 19) was calculated by the rule of mixtures and the $C_{p}$ of water, and the $\rho$ was corrected with respect to the $w_{H 2 O}$ and the volume shrinkage. The volume shrinkage was estimated by measuring the dimensions of the foams after each RH conditioning cycle.

$$
C_{P_{\text {wet }}}=\left(1-w_{\mathrm{H} 2 \mathrm{O}}\right) \cdot C_{P_{\text {dry }}}+C_{P_{\mathrm{H}_{2} \mathrm{O}}} \cdot w_{\mathrm{H} 2 \mathrm{O}}
$$

where $w_{H 2 O}$ is the water content in the foams in mass fraction, $C p_{d r y}$ is the dry specific heat capacity at constant pressure of the foams measured in the DSC in $\mathrm{J} \mathrm{kg}^{-1} \mathrm{~K}^{-1}$ and $C p_{\mathrm{H} 2 \mathrm{O}}$ is the specific heat capacity at constant pressure of water in $\mathrm{J} \mathrm{kg}^{-1} \mathrm{~K}^{-1}$.

Moisture uptake. The water vapor sorption of the CNC foams under controlled RH and T was determined by measuring the weight change using a high-precision balance (BP 210 S, Sartorius, Germany) placed inside a humidity chamber as described previously ${ }^{25}$. Prior to the measurements, the foams were dried at $313 \mathrm{~K}$ and $20 \% \mathrm{RH}$. The moisture content $\left(w_{\mathrm{H} 2 \mathrm{O}}\right)$ as a function of $R H(20,35,50,65$ and $80 \%)$ was assessed at $295 \mathrm{~K}$. Each measurement lasted $6 \mathrm{~h}$ to ensure that steady state was reached, and the foam mass was measured every $5 \mathrm{~min}$.

Thermal conductivity measurement. The thermal conductivities $\left(\lambda, \mathrm{mW} \mathrm{m}^{-1} \mathrm{~K}^{-1}\right)$ of the foams were determined using the TPS 2500 S Hot Disk Thermal Constants Analyzer in the anisotropic mode. The TPS 2500 $S$ Hot Disk Thermal Constants Analyzer has a reported accuracy for isotropic materials of $2-5 \%{ }^{44}$ but the accuracy is less for anisotropic, low thermal conductivity materials ${ }^{27}$. The Kapton transient plane sensor $(3.2 \mathrm{~mm}$ in radius) was placed between two identical foam pieces (diameter: $4.1 \pm 0.1 \mathrm{~cm}$; height: $2.4 \pm 0.2 \mathrm{~cm}$ ). Good thermal contact between the sensor and the foams was ensured by putting a small weight ( $39 \mathrm{~g})$ onto the samples resulting in a contact pressure of $339 \mathrm{~N} \mathrm{~m}^{2} \pm 0.4^{45}$. The heating power and the measurement time were $10 \mathrm{~mW}$ and $5 \mathrm{~s}$ for $\mathrm{CNC}_{25-40}$ and $10 \mathrm{~mW}$ and $10 \mathrm{~s}$ for $\mathrm{CNC}_{50-130}$, respectively. The foams were enclosed in a customized cell, allowing the $R H$ to be controlled $(2-80 \% R H)^{25}$. Five independent measurements were performed at $15-\mathrm{min}$ intervals for each $R H$ at $295 \mathrm{~K}$ and each foam pair and every foam was investigated using 2-4 different pair of foams with the same density. The TPS method in the anisotropic mode yields the radial thermal diffusivity (see Supplementary Fig. S3), from which the thermal conductivity is calculated by multiplying with the volumetric heat capacity $\left(\rho_{a p p} C_{p}\right)$. The axial thermal conductivity of the anisotropic foams at different $R H$ and $T$ were obtained using the software in the Hot Disk as previously described ${ }^{16}$.

The foam shrinkage during thermal conductivity experiments was taken into consideration when assessing the volume change of the foams during the water uptake measurements. The foams were mounted on the customized cell and the volume of the foams was measured after each humidity cycle with a caliper. Taking into consideration the volume shrinkage and the moisture content, the wet density of the foams was calculated and used for the calculations of the thermal conductivity in Hot Disk.

\section{Data availability}

The datasets generated during and/or analysed during the current study are available in the [Materials Cloud] repository, [https://doi.org/10.24435/materialscloud:49-t3].

Received: 18 February 2021; Accepted: 26 August 2021

Published online: 21 September 2021

\section{References}

1. Budtova, T. Bio-based aerogels : A new generation of thermal superinsulating materials. in Cellulose science and technology: Chemistry, analysis and applications (eds. Rosenau, T., Potthast, A. \& Hell, J.) 371-392 (Wiley, 2019). https://doi.org/10.1002/9781119217 619.ch15

2. Duong, H. M. \& Nguyen, S. T. Nanocellulose aerogels as thermal insulation materials. in Nano and biotech based materials for energy building efficiency (eds. Pacheco Torgal, F., Buratti, C., Kalaiselvam, S., Granqvist, C. \& Ivanov, V.) 411-427 (Springer, Cham, 2016). https://doi.org/10.1007/978-3-319-27505-5_15

3. Lavoine, N. \& Bergström, L. Nanocellulose-based foams and aerogels: Processing, properties, and applications. J. Mater. Chem. A 5, 16105 (2017).

4. Federation of European Rigid Polyurethane Foam Association. Thermal insulation materials made of rigid polyurethane foam (PUR/ PIR). (2006).

5. Winterling, H. \& Sonntag, N. Rigid polystyrene foam (EPS, XPS). Kunststoffe International 101 (2011).

6. Churkina, G. et al. Buildings as a global carbon sink. Nat. Sustain. 3, 269-276 (2020).

7. Klemm, D. et al. Nanocelluloses: A new family of nature-based materials. Angew. Chemie - Int. Ed. 50, 5438-5466 (2011).

8. Diaz, J. A. et al. Thermal conductivity in nanostructured films: From single cellulose nanocrystals to bulk films. Biomacromol 15, 4096-4101 (2014).

9. Sato, K., Tominaga, Y. \& Imai, Y. Nanocelluloses and related materials applicable in thermal management of electronic devices: A review. Nanomaterials 10, 1-13 (2020). 
10. Jelle, B. P. Traditional, state-of-the-art and future thermal building insulation materials and solutions-Properties, requirements and possibilities. Energy Build. 43, 2549-2563 (2011).

11. Apostolopoulou-Kalkavoura, V., Munier, P. \& Bergström, L. Thermally insulating nanocellulose-based materials. Adv. Mater. 200, 2020 (1839).

12. Sakai, K., Kobayashi, Y., Saito, T. \& Isogai, A. Partitioned airs at microscale and nanoscale: Thermal diffusivity in ultrahigh porosity solids of nanocellulose. Sci. Rep. 6, 20434 (2016).

13. Kobayashi, Y., Saito, T. \& Isogai, A. Aerogels with 3D ordered nanofiber skeletons of liquid-crystalline nanocellulose derivatives as tough and transparent insulators. Angew. Chemie Int. Ed. 53, 10394-10397 (2014).

14. Jiménez-Saelices, C., Seantier, B., Cathala, B. \& Grohens, Y. Spray freeze-dried nanofibrillated cellulose aerogels with thermal superinsulating properties. Carbohydr. Polym. 157, 105-113 (2017).

15. Song, M., Jiang, J., Qin, H., Ren, X. \& Jiang, F. Flexible and super thermal-insulating cellulose nanofibrils/emulsion composite aerogel with quasi-closed pores. ACS Appl. Mater. Interfaces 12, 45363-45372 (2020).

16. Apostolopoulou-Kalkavoura, V. et al. Humidity-dependent thermal boundary conductance controls heat transport of superinsulating nanofibrillar foams humidity-dependent thermal boundary conductance controls heat transport of super-insulating nanofibrillar foams. Matter https://doi.org/10.1016/j.matt.2020.11.007 (2020).

17. Jiménez-Saelices, C., Seantier, B., Grohens, Y. \& Capron, I. Thermal superinsulating materials made from nanofibrillated cellulosestabilized pickering emulsions. ACS Appl. Mater. Interfaces 10, 16193-16202 (2018).

18. Seantier, B., Bendahou, D., Bendahou, A., Grohens, Y. \& Kaddami, H. Multi-scale cellulose based new bio-aerogel composites with thermal super-insulating and tunable mechanical properties. Carbohydr. Polym. 138, 335-348 (2016).

19. Arcari, M. et al. Structure property relationships of cellulose nanofibril hydro- and aerogels and their building blocks. Nanoscale 12, 11638-11646 (2020).

20. Munier, P., Gordeyeva, K., Bergström, L. \& Fall, A. B. Directional freezing of nanocellulose dispersions aligns the rod-like particles and produces low-density and robust particle networks. Biomacromol 17, 1875-1881 (2016).

21. Reid, M. S., Villalobos, M. \& Cranston, E. D. benchmarking cellulose nanocrystals: From the laboratory to industrial production. Langmuir 33, 1583-1598 (2017).

22. Abitbol, T. \& Cranston, E. D. Chiral Nematic Self-Assembly of cellulose nanocrystals in suspensions and solid films. in Handbook of green materials, Vol. 3: Self- and direct- assembling of bionanomaterials 37-56 (World Scientific, 2014). https://doi.org/10.1142/ 9789814566469_0035

23. Schütz, C. et al. From equilibrium liquid crystal formation and kinetic arrest to photonic bandgap films using suspensions of cellulose nanocrystals. Curr. Comput.-Aided Drug Des. 10, 199 (2020).

24. Munier, P., Apostolopoulou-Kalkavoura, V., Persson, M. \& Bergström, L. Strong silica-nanocellulose anisotropic composite foams combine low thermal conductivity and low moisture uptake. Cellulose 27, 10825-10836 (2019).

25. Apostolopoulou-Kalkavoura, V., Gordeyeva, K., Lavoine, N. \& Bergström, L. Thermal conductivity of hygroscopic foams based on cellulose nanofibrils and a nonionic polyoxamer. Cellulose 25, 1117-1126 (2018).

26. White, G. H. Basics of estimating measurement uncertainty. Clin. Biochem. Rev. 29(Suppl I), S53-60 (2008).

27. Trofimov, A. A., Atchley, J., Shrestha, S. S., Desjarlais, A. O. \& Wang, H. Evaluation of measuring thermal conductivity of isotropic and anisotropic thermally insulating materials by transient plane source (Hot Disk) technique. J. Porous Mater. 27, 1791-1800 (2020).

28. Smith, D. S. et al. Thermal conductivity of porous materials. J. Mater. Res. 28, 2260-2272 (2013).

29. Liang, X. G. \& Qu, W. Effective thermal conductivity of gas-solid composite materials and the temperature difference effect at high temperature. Int. J. Heat Mass Transf. 42, 1885-1893 (1999).

30. Staggs, J. E. J. Estimating the thermal conductivity of chars and porous residues using thermal resistor networks. Fire Saf. J. 37, $107-119$ (2002).

31. Ochs, F., Heidemann, W. \& Müller-Steinhagen, H. Effective thermal conductivity of moistened insulation materials as a function of temperature. Int. J. Heat Mass Transf. 51, 539-552 (2008).

32. Wang, M., Wang, J., Pan, N., Chen, S. \& He, J. Three-dimensional effect on the effective thermal conductivity of porous media. J. Phys. D. Appl. Phys. 40, 260-265 (2007).

33. Carson, J. K., Lovatt, S. J., Tanner, D. J. \& Cleland, A. C. Predicting the effective thermal conductivity of unfrozen, porous foods. J. Food Eng. 75, 297-307 (2006).

34. Zeng, S. Q., Hunt, A. \& Greif, R. Transport properties of gas in silica aerogel. J. Non. Cryst. Solids 186, 264-270 (1995).

35. Han, Z. \& Fina, A. Thermal conductivity of carbon nanotubes and their polymer nanocomposites: A review. Prog. Polym. Sci. 36, 914-944 (2011).

36. Adachi, K. et al. Thermal conduction through individual cellulose nanofibers. Appl. Phys. Lett. 118, 053701 (2021).

37. Chernatynskiy, A., Clarke, D. R. \& Phillpot, S. R. Thermal transport in nanostructured materials. in Handbook of nanoscience, engineering, and technology (eds. Goddard III, W., Brenner, D., Lyshevski, S. \& Iafrate, G.) 568-595 (CRC Press, 2018). https://doi. org/10.1201/b11930-24

38. Wicklein, B. et al. Thermally insulating and fire-retardant lightweight anisotropic foams based on nanocellulose and graphene oxide. Nat. Nanotechnol. 10, 277-283 (2015).

39. Scandinavian pulp paper and board testing committee. Total acidic group content. Scandinavian pulp, paper and board testing committee 1-4 (2002).

40. Kriechbaum, K., Munier, P., Apostolopoulou-Kalkavoura, V. \& Lavoine, N. Analysis of the porous architecture and properties of anisotropic nanocellulose foams: A novel approach to assess the quality of cellulose nanofibrils (CNFs). ACS Sustain. Chem. Eng. 6, 11959-11967 (2018).

41. Baisheng, N., Xianfeng, L., Longlong, Y., Junqing, M. \& Xiangchun, L. Pore structure characterization of different rank coals using gas adsorption and scanning electron microscopy. Fuel 158, 908-917 (2015).

42. Segal, L., Creely, J. J., Martin, A. E. \& Conrad, C. M. An empirical method for estimating the degree of crystallinity of native cellulose using the X-ray diffractometer. Text. Res. J. 29, 786-794 (1959).

43. Haywood, A. D. et al. New insights into the flow and microstructural relaxation behavior of biphasic cellulose nanocrystal dispersions from RheoSANS. Soft Matter 13, 8451-8462 (2017).

44. Hot Disk TPS 2500 S. Available at: https://www.hotdiskinstruments.com/products-services/instruments/tps-2500-s/.

45. Gustafsson, S. E. Transient plane source techniques for thermal conductivity and thermal diffusivity measurements of solid materials. Rev. Sci. Instrum. 62, 797-804 (1991).

\section{Acknowledgements}

We thank Alisa Gordeeva for running the powder XRD measurements for the determination of the crystallinity index, Konstantin Kriechbaum for assisting with AFM imaging and fruitful discussions, Ehsan Hadi for assisting with SEM imaging and Carina Schiele for performing DSC measurements. The authors acknowledge financial support from the Wallenberg Wood Science Center (WWSC) and the Swedish Energy Agency (Energimyndigheten, project 2019-006749). 


\section{Author contributions}

V.A.K. took part in the conception of the experiments, all aspects of the sample preparation and characterization, and the production of the manuscript. P.M. took part in all aspects of the sample preparation and characterization, and the production of the manuscript. L.D. took part in the sample preparation and SEM imaging. V.L.H. took part in the sample preparation, titration, SEM imaging, SEM image analysis and XRD analysis. L.B. took part in the conception of the experiments and the production of the manuscript.

\section{Funding}

Open access funding provided by Stockholm University.

\section{Competing interests}

The authors declare no competing interests.

\section{Additional information}

Supplementary Information The online version contains supplementary material available at https://doi.org/ 10.1038/s41598-021-98048-y.

Correspondence and requests for materials should be addressed to L.B.

Reprints and permissions information is available at www.nature.com/reprints.

Publisher's note Springer Nature remains neutral with regard to jurisdictional claims in published maps and institutional affiliations.

(c) (i) Open Access This article is licensed under a Creative Commons Attribution 4.0 International

License, which permits use, sharing, adaptation, distribution and reproduction in any medium or format, as long as you give appropriate credit to the original author(s) and the source, provide a link to the Creative Commons licence, and indicate if changes were made. The images or other third party material in this article are included in the article's Creative Commons licence, unless indicated otherwise in a credit line to the material. If material is not included in the article's Creative Commons licence and your intended use is not permitted by statutory regulation or exceeds the permitted use, you will need to obtain permission directly from the copyright holder. To view a copy of this licence, visit http://creativecommons.org/licenses/by/4.0/.

(C) The Author(s) 2021 